\title{
Bibliometric analysis of the journal "Campos en ciencias sociales", from the fields of action of the Santo Tomás University (Colombia)
}

\section{Laura Alejandra Urrego Gaitán, Comunicadora Social laura.urrego@usantotomas.edu.co}

\section{Miguel Urra Canales, Trabajador Social y Sociólogo dec.sociologia@usantotomas.edu.co}

\begin{abstract}
:
This article addresses the alignment of the journal "Campos en ciencias sociales" with the fields of action declared by the university that publishes it. For this, a bibliometric analysis has been carried out, based on the 102 articles published in 8 volumes between 2013 and 2020 . As results, an overwhelming proportion of texts focused on the field "Society" and a still incipient presence of the "Environment" field or a joint work of both fields.
\end{abstract}

Key words: fields of action, journal, social sciences. 


\section{PLANTEAMIENTO}

Between 2012 and 2018, the Universidad Santo Tomás carried out a work of information systematization, with the aim of defining its national goals and identifying its fields of action, taking into account its capacities and institutional strengths in teaching, research and social projection of the different Academic programs in Headquarters and Sectionals. This work was led by the General Academic Vice-rectory, together with the Units for Curriculum Development and Teacher Training, Research and University Social Responsibility. (Ostos, O.L. and Cortés, M.A. 2018).

In the institutional documents of the USTA, such as the Development Plan 2012-2015 or the Comprehensive Multicampus Plan 2016-2027, it is firmly committed to concentrating efforts and focusing projects in some specific areas, in order to multiply the impacts. As a consequence of this, the fields of action were defined as "areas or spaces of significant reality, with relative limits, where substantive functions converge from rigorous investigative processes, in relation to the problems that have been identified and that are of interest to the USTA, with evidence of context, experience and interdisciplinarity "(Cedeño, 2016).

Finally, after a long process of analysis and deliberations, two major fields of action were defined: society and the environment. Each of them includes a series of interactions, that is, areas where the USTA has shown greater intellectual and academic production. They are as follows (Ostos, O.L. and Cortés, M.A. 2018: 36):

\begin{tabular}{|l|l|}
\hline SOCIETY & ENVIRONMENT \\
\hline 1. Communication & 1. Environments \\
\hline 2. Economic and social development & 2. Biodiversity \\
\hline 3. Public Policy & 3. Habitat \\
\hline 4. Human rights & 4. Ecology \\
\hline 5. Economy & 5. Atmosphere \\
\hline 6. Information literacy & 6. Aquatic ecosystems \\
\hline 7. Knowledge management & 7. Terrestrial ecosystems \\
\hline 8. Social behavior & 8. Effects of human activities \\
\hline 9. Social systems & 9. Environmental management \\
\hline 10. Health, Well-being and public health & 10. Contamination \\
\hline 11. Social groups & 11. Clean and sustainable energies \\
\hline 12. Value systems & 12. Sustainable cities \\
\hline 13. Education & 13. Smart cities \\
\hline 14. Infrastructure & 14. Responsible production and \\
\hline 15. Urban planning & consumption \\
\hline 16. Information users & \\
\hline 17. Peace and coexistence & \\
\hline 18. Citizen participation & \\
\hline 19. Scientific developments applied to the & \\
transformation of society & \\
\hline 20. Equality and justice & \\
\hline
\end{tabular}

As for the journal "Campos en Ciencias Sociales", as expressed on its website (https://revistas.usantotomas.edu.co/index.php/campos/index): "it is an interdisciplinary publication in the social sciences, of a scientific and investigative nature, which was born in 2013 and aims to offer the academic community a space for debate and dissemination about the social sciences from the knowledge society, which influences different areas such as cultural, social , political and economic". 
It is a joint editorial project of the faculties of Sociology, Graphic Design and Social Communication, whose thematic axes are territories (understanding them as not only geographical but also virtual spatialities), culture, counterculture, identities and social movements (peasants, indigenous, Afro, feminists, among others) and visual communication and cultural products (cinema, television, literature).

In formal terms, it has a semi-annual periodicity, its ISSN is 2339-3688, its electronic ISSN is 2500-6681 and its DOI is 10.15332 / 25006681. At the time of publishing this article, 102 articles have passed through its pages and it is indexed in Redib, Clase, Circ, Base, Google Scholar, Miar, Ulrich's Periodical Directory, EZB, LatinRev, Erih Plus, Seriunam, Actualidad Iberoamericana, Cite Factor and Academic Resource Index.

With these definitions, this article aims to analyze the alignment of the articles published in the Campos magazine with these fields of action and institutional interactions of the Santo Tomás University. This work will help evaluate the magazine's strengths and weaknesses and project editorial strategies for the future.

\section{METHODOLOGY}

In order to carry out the analysis that was carried out during the writing of this article, we first sought to review all the texts published in the magazine Campos en Ciencias Sociales since its first issue in 2013, under the title " Community, action and communication ", which sought to" define the object of study of a magazine from the social sciences, which is a very complex task, insofar as this field of knowledge is made up of a network of disciplines, approaches, debates and meeting spaces ". (Castiblanco, 2013); up to the number of volume 8 published for the year 2019, which is nested under the work proposal in relation to lines on identities and social conflicts in the world, and that in the words of its invited editor Miguel urra Canales, "intends to open a space to peace within the studies of the sociology of conflict (...) in relation to the social research carried out on this subject in its multiple facets: agreements, postagreement, victims, ex-combatants, peace pedagogy and public policies "(page 9,2020 ). Adding a total of 102 articles, in 8 volumes and 15 numbers, with an average of 12.7 articles per volume and 6.8 per number, respectively.

In this way, we start from the definition of fields of action worked at the Santo Tomás University, as "areas or spaces of significant reality, with relative limits, where substantive functions converge from rigorous investigative processes, in relation to the problems (...) that are of interest to the USTA, with evidence of context, experience and interdisciplinarity "(Ostos and Cortés, 2018), which allows the consolidation of a categorical group that to identify the intellectual production of the Campos magazine in relation to its articles and the scientific production expressed in them. 


\section{RESULTS}

First, a classification of the fields was made in relation to each published article (excluding the editorials and presentations of each number), in order to identify the alignment of the journal's products with the fields of action proposed by the research guidelines. of the institution, to evaluate the points of coincidence of the contents, with the aim of consolidating future strategies that allow, not only the exploration of new topics, but in turn the expansion of the academic horizons that are worked on in these stories, agreeing with the dissemination positions of this scientific journal. (See table 1)

\section{Tabla 1: Classification of each article.}

\begin{tabular}{|c|c|c|c|c|c|}
\hline $\mathbf{V}$ & $\mathbf{N}$ & ARTICLE & AUTHORS & YEAR & FIELDS \\
\hline 1 & 1 & $\begin{array}{l}\text { Las organizaciones comunitarias como } \\
\text { instituciones intermedias: las Juntas de Acción } \\
\text { Comunal en el municipio de Tenjo }\end{array}$ & Martha Milena Bautista Gómez & 2013 & Society \\
\hline 1 & 1 & $\begin{array}{l}\text { Entre la participación ciudadana y las } \\
\text { resistencias creativas: Una experiencia en la } \\
\text { localidad de Engativá }\end{array}$ & Liliana Camargo Urrea & 2013 & Society \\
\hline 1 & 1 & $\begin{array}{l}\text { Espacios comunicativos para la convivencia y la } \\
\text { participación. Pensamiento y práctica desde la } \\
\qquad \text { IAP }\end{array}$ & Richard Ducón Salas & 2013 & Society \\
\hline 1 & 1 & $\begin{array}{l}\text { Lo comunitario en las radios comunitarias: } \\
\text { sentidos en juego }\end{array}$ & Sandra Liliana Osses Rivera & 2013 & Society \\
\hline 1 & 1 & $\begin{array}{c}\text { Modelo de televisión comunitaria para el } \\
\text { desarrollo humano: estudio de la televisión } \\
\text { comunitaria de Colombia y nueva propuesta de } \\
\text { programación para su audiencia }\end{array}$ & Lizandro Angulo Rincón & 2013 & Society \\
\hline 1 & 1 & $\begin{array}{c}\text { Protestar por los derechos, el derecho a } \\
\text { protestar. Una lectura desde las audiencias }\end{array}$ & Silvina Berti, Ariadna Cantú & 2013 & Society \\
\hline 1 & 1 & $\begin{array}{c}\text { Del ejercicio de la prostitución al ejercicio del } \\
\text { trabajo sexual como forma de reivindicar } \\
\text { derechos negados }\end{array}$ & $\begin{array}{l}\text { Carlos Alfonso Laverde } \\
\text { Rodríguez }\end{array}$ & 2013 & Society \\
\hline 1 & 1 & Sobre mujeres y desplazamiento & Olga Lucía Perdomo & 2013 & Society \\
\hline 1 & 1 & $\begin{array}{l}\text { In memoriam: La plataforma massmediática } \\
\text { como dispositivo de emancipación }\end{array}$ & Liliana Rocío Torres López & 2013 & Society \\
\hline 1 & 2 & $\begin{array}{c}\text { Mercado y consumo: economía política de las } \\
\text { telecomunicaciones en Colombia }\end{array}$ & Ancízar Narváez Montoya & 2013 & Society \\
\hline 1 & 2 & $\begin{array}{l}\text { El manejo informativo del desplazamiento } \\
\text { forzado en dos medios electrónicos } \\
\text { colombianos: eltiempo.com y semana.com }\end{array}$ & Alejandra Salamanca Rodríguez & 2013 & Society \\
\hline 1 & 2 & $\begin{array}{l}\text { La construcción discursiva de un genocidio en } \\
\text { Colombia: una aproximación a la versión de las } \\
\text { Fuerzas Militares en el caso de la Unión } \\
\text { Patriótica }\end{array}$ & $\begin{array}{l}\text { Liliana A. Silva Bello, Mauricio } \\
\text { Poveda Pineda }\end{array}$ & 2013 & Society \\
\hline 1 & 2 & $\begin{array}{l}\text { Patrimonio cultural y turismo en San Pedro de } \\
\text { Atacama, Chile }\end{array}$ & Andrea Hurtado Quiñones & 2013 & Environment \\
\hline 1 & 2 & $\begin{array}{c}\text { A la zaga de una propuesta para la combinación } \\
\text { de metodologías de investigación }\end{array}$ & $\begin{array}{l}\text { María Victoria Rugeles Gélvez, } \\
\text { Eliana Del Rosario Herrera } \\
\text { Huérfano, Carlos Andrés Muñoz } \\
\text { Sandoval }\end{array}$ & 2013 & Society \\
\hline 1 & 2 & $\begin{array}{c}\text { El uso de la imagen como herramienta de } \\
\text { investigación }\end{array}$ & Mónica Eliana García Gil & 2013 & Society \\
\hline 1 & 2 & Doscientos años de un periódico no tan feliz & Jaime Alberto Rojas Rodríguez & 2013 & Society \\
\hline 1 & 2 & $\begin{array}{c}\text { Memoria, prácticas artísticas y espacio público: } \\
\text { posibilidades frente al conflicto armado } \\
\text { colombiano }\end{array}$ & Santiago Jiménez Mojica & 2013 & $\begin{array}{l}\text { Society and } \\
\text { Environment }\end{array}$ \\
\hline 1 & 2 & Hermenéutica de los cuerpos & Constanza Gómez Gavilán & 2013 & Environment \\
\hline
\end{tabular}


Archivo, memoria y dictadura: activistas

21 religiosos y enfrentamiento al régimen dictatorial João Marcus Figuereido Assis 2014 Society en Brasill

\begin{tabular}{|c|c|c|c|c|c|}
\hline 2 & 1 & $\begin{array}{l}\text { Historia, memoria y olvido: la historia contada } \\
\text { sobre el levantamiento de } 1935 \text { en el Partido } \\
\text { Comunista de Brasil (1943-1958) }\end{array}$ & Jayme Fernandes Ribeiro & 2014 & Society \\
\hline 2 & 1 & $\begin{array}{l}\text { Memorias de la secularización: el registro de las } \\
\text { transformaciones en el escenario religioso } \\
\text { brasilero a través de las estadísticas de } \\
\text { individuos identificados como sin religión }\end{array}$ & Denise dos Santos Rodrigues & 2014 & Society \\
\hline 2 & 1 & $\begin{array}{l}\text { Sacralidad, tormento y rescate: la vida social de } \\
\text { la chicha muisca }\end{array}$ & Pablo Felipe Gómez-Montañez & 2014 & Environment \\
\hline 2 & 1 & Historia oral: ¿una historia popular? & $\begin{array}{c}\text { Pedro Rodríguez Rojas, Janette } \\
\text { García Yépez }\end{array}$ & 2014 & Society \\
\hline 2 & 1 & $\begin{array}{l}\text { Reseña bibliográfica: Cartel ilustrado en } \\
\text { Colombia: década 1930-1940 }\end{array}$ & Jesús Alfonso Gallardo Vega & 2014 & Society \\
\hline 2 & 2 & $\begin{array}{l}\text { La Hacienda El Carmen como escenario } \\
\text { coyuntural de disputa sobre memoria, territorio y } \\
\text { patrimonio }\end{array}$ & Andrea Castro Bernal & 2014 & $\begin{array}{l}\text { Society and } \\
\text { Environment }\end{array}$ \\
\hline 2 & 2 & $\begin{array}{l}\text { Lecciones de memoria. El recuerdo de } 1971 \text { en } \\
\text { las protestas estudiantiles de } 2011 \text { en Colombia }\end{array}$ & Edwin Cruz Rodríguez & 2014 & Society \\
\hline 2 & 2 & $\begin{array}{l}\text { Una mirada a la cátedra de estudios } \\
\text { afrocolombianos a partir de las reflexiones de los } \\
\text { líderes de la Red Elegguá en la ciudad de } \\
\text { Bogotá }\end{array}$ & Ana Cristina Sotelo Manrique & 2014 & Society \\
\hline 2 & 2 & $\begin{array}{c}\text { Patrimonio y memoria: encuentros y } \\
\text { desencuentros por el hallazgo del cementerio } \\
\text { muisca en la hacienda El Carmen, en Bogotá, D. } \\
\text { C. }\end{array}$ & $\begin{array}{l}\text { Angie Tatiana Cardozo } \\
\text { Rodríguez }\end{array}$ & 2014 & $\begin{array}{l}\text { Society and } \\
\text { Environment }\end{array}$ \\
\hline 2 & 2 & En busca de la alteridad & César Augusto Muñoz Marín & 2014 & Society \\
\hline 2 & 2 & $\begin{array}{l}\text { Bibliographic review: Emergencies of the territory } \\
\text { and local communication. Experiences of } \\
\text { communication and development about the } \\
\text { environment in Colombia }\end{array}$ & $\begin{array}{l}\text { Fredy Leonardo Reyes } \\
\text { Albarracín }\end{array}$ & 2014 & Society \\
\hline 3 & 1 & $\begin{array}{l}\text { Los sistemas productivos tradicionales y el } \\
\text { programa RESA en el resguardo Ticoya de } \\
\text { Puerto Nariño }\end{array}$ & Juan José Vieco & 2015 & Environment \\
\hline 3 & 1 & $\begin{array}{l}\text { La narrativa como herramienta didáctica y de } \\
\text { comunicación para la enseñanza de la historia } \\
\text { social contemporánea y reciente }\end{array}$ & $\begin{array}{l}\text { Renée Isabel Mengo, Pablo } \\
\text { Rubén Tenaglia }\end{array}$ & 2015 & Society \\
\hline 3 & 1 & $\begin{array}{l}\text { Comunicación para el diálogo político e } \\
\text { intercultural. Derecho a la comunicación y } \\
\text { ciudadanía comunicacional }\end{array}$ & Washington Uranga & 2015 & Society \\
\hline 3 & 1 & $\begin{array}{l}\text { Desarrollo e hiperconsumo: la producción de lo } \\
\text { efímero }\end{array}$ & François -Xavier Tinel & 2015 & Society \\
\hline 3 & 1 & $\begin{array}{l}\text { La dinámica social de las orientaciones de } \\
\text { desarrollo en América Latina }\end{array}$ & François Houtart & 2015 & Society \\
\hline 3 & 2 & $\begin{array}{l}\text { Jóvenes y representaciones sociales sobre la } \\
\text { foto de perfil en Facebook }\end{array}$ & Adriana Rubiano Rubiano & 2015 & Society \\
\hline 3 & 2 & $\begin{array}{l}\text { Aproximación al estudio de las formas de } \\
\text { representación de los actores armados en } \\
\text { justicia y paz }\end{array}$ & María Teresa Suárez González & 2015 & Society \\
\hline 3 & 2 & $\begin{array}{l}\text { La co-creación como plataforma estratégica para } \\
\text { reposicionar la marca ciudad. Caso de estudio: } \\
\text { Medellín, ciudad innovadora. }\end{array}$ & $\begin{array}{l}\text { Daniela Alejandra Gordillo } \\
\text { Rojas }\end{array}$ & 2015 & Society \\
\hline 3 & 2 & $\begin{array}{l}\text { Comportamiento humano y prevención de } \\
\text { dengue: Estudio en barrios endémicos de } \\
\text { Barranquilla, Bucaramanga y Armenia } \\
\text { (Colombia) }\end{array}$ & $\begin{array}{l}\text { Giannina Torres Pérez, Daniel } \\
\text { Ernesto Aguilar Rodríguez }\end{array}$ & 2015 & Environment \\
\hline
\end{tabular}




\begin{tabular}{|c|c|c|c|c|c|}
\hline 3 & 2 & $\begin{array}{l}\text { Participación de los padres de familia en la } \\
\text { escuela. La violencia como mediadora de la } \\
\text { relación entre los padres y la escuela }\end{array}$ & Lina Paola Martínez Nieto & 2015 & Society \\
\hline 3 & 2 & $\begin{array}{c}\text { La planeación en el municipio y resguardo de } \\
\text { Jambaló (Colombia) }\end{array}$ & Máryuri Castaño Canoas & 2015 & Environment \\
\hline 3 & 2 & $\begin{array}{l}\text { A propósito de los "falsos opuestos" en Ciencias } \\
\text { Sociales -La IAP como posibilidad para la lectura } \\
\text { integral y crítica de la realidad- }\end{array}$ & Esther Gutiérrez Mora & 2015 & Society \\
\hline 4 & 1 & $\begin{array}{c}\text { Cine, poder e historia: la representación y } \\
\text { construcción social del indígena en el cine } \\
\text { ficción venezolano durante la década de los } \\
\text { años } 80\end{array}$ & Fidel Rodríguez Velásquez & 2016 & Society \\
\hline 4 & 1 & $\begin{array}{c}\text { La comunicación visual a través de Pepe Mexía } \\
\text { y Ricardo Rendón, 1915-1930 }\end{array}$ & $\begin{array}{l}\text { Arcadio Alexander Aldana } \\
\text { Rincón }\end{array}$ & 2016 & Society \\
\hline 4 & 1 & $\begin{array}{c}\text { Catedrales como bibliotecas: filosofía, literatura y } \\
\text { espiritualidad }\end{array}$ & $\begin{array}{c}\text { Juan Sebastián Ballén } \\
\text { Rodríguez }\end{array}$ & 2016 & Society \\
\hline 4 & 1 & $\begin{array}{l}\text { Los imaginarios sociales desde Armando Silva, } \\
\text { sus avances, transformaciones y productos }\end{array}$ & $\begin{array}{l}\text { Camila Contreras Parada, } \\
\text { Cristhian Uribe Mendoza, Felipe } \\
\text { Aliaga Sáez }\end{array}$ & 2016 & Society \\
\hline 4 & 1 & $\begin{array}{l}\text { Comunicación y deporte: un campo integrador } \\
\text { para el análisis del fenómeno deportivo }\end{array}$ & Lizandro Angulo Rincón & 2016 & Society \\
\hline 4 & 2 & $\begin{array}{l}\text { La memoria como eje de cambio social en la } \\
\text { escuela }\end{array}$ & Angélica María Valencia Murillo & 2016 & Society \\
\hline 4 & 2 & $\begin{array}{l}\text { Heraldos de cine: imágenes del cine y la } \\
\text { construcción estética del colombiano moderno }\end{array}$ & $\begin{array}{l}\text { Claudia Angélica Reyes } \\
\text { Sarmiento }\end{array}$ & 2016 & Society \\
\hline 4 & 2 & $\begin{array}{c}\text { Muy bonito todo. Investigar imágenes y objetos } \\
\text { contemporáneos desde la cultura mediática y la } \\
\text { cultura popular }\end{array}$ & Edward Salazar & 2016 & Society \\
\hline 4 & 2 & $\begin{array}{l}\text { Prácticas sociales, creatividad y habitar en las } \\
\text { regiones Medellín, Antioquia (Colombia) }\end{array}$ & María Ginette Múnera Barrios & 2016 & Environment \\
\hline 5 & $\begin{array}{l}1 \\
y \\
2\end{array}$ & $\begin{array}{l}\text { Periodismo regional y conflictos sociales. } \\
\text { Análisis de las opiniones de los diarios Correo y } \\
\text { Los Andes de Puno (Perú) durante el conflicto } \\
\text { social del "Aimarazo" (2011) }\end{array}$ & Miguel Angel Angulo Giraldo & 2017 & Society \\
\hline 5 & $\begin{array}{l}1 \\
y \\
2\end{array}$ & $\begin{array}{l}\text { Economía de la coca y violencia: realidades } \\
\text { desde el Corregimiento de El Plateado, } \\
\text { Municipio de Argelia, Cauca (Colombia) }\end{array}$ & Denis Antonio Arboleda Suárez & 2017 & Society \\
\hline 5 & $\begin{array}{l}1 \\
y \\
2\end{array}$ & $\begin{array}{l}\text { Constitución y ampliación de resguardos } \\
\text { indígenas en Colombia. Una mirada al avance } \\
\text { histórico y perspectivas de cumplimiento }\end{array}$ & Oscar Andrés Garzón Zabala & 2017 & Environment \\
\hline 5 & $\begin{array}{l}1 \\
y \\
2\end{array}$ & $\begin{array}{c}\text { Aproximación a las prácticas ciudadanas en la } \\
\text { escuela: el caso del colegio Fernando González } \\
\text { Ochoa de la localidad de Usme (Bogotá, } \\
\text { Colombia) }\end{array}$ & Rodrigo Gómez & 2017 & Society \\
\hline 5 & $\begin{array}{l}1 \\
y \\
2\end{array}$ & $\begin{array}{c}\text { El conflicto, la guerra y la paz. Una aproximación } \\
\text { desde la perspectiva del realismo político en } \\
\text { Nicolás Maquiavelo }\end{array}$ & Víctor Alfonso Londoño Villegas & 2017 & Society \\
\hline 5 & $\begin{array}{l}1 \\
y \\
2\end{array}$ & $\begin{array}{l}\text { Voces testimoniales y realidad experimental en } \\
\text { Guadalupe años sin cuenta del grupo la } \\
\text { Candelaria }\end{array}$ & Carlos Augusto Rojas Muñoz & 2017 & Society \\
\hline 5 & $\begin{array}{l}1 \\
y \\
2\end{array}$ & $\begin{array}{l}\text { Un acercamiento al patrimonio cultural inmaterial } \\
\text { su salvaguarda y patrimonialización }\end{array}$ & $\begin{array}{l}\text { Montserrat Patricia Rebollo } \\
\text { Cruz }\end{array}$ & 2017 & Society \\
\hline 6 & 1 & $\begin{array}{c}\text { Redes que se construyen dentro de la } \\
\text { comunidad indígena Embera desplazada en } \\
\text { Bogotá (Colombia): caso del alojamiento Embera } \\
\text { en Santafé }\end{array}$ & María Camila Sánchez Delgado & 2018 & Society \\
\hline 6 & 1 & $\begin{array}{l}\text { Políticas de habitabilidad en calle en Bogotá, } \\
\text { Colombia ¿hacia el desarrollo humano integral? }\end{array}$ & Luisa Fernanda Zamudio Rocha & 2018 & $\begin{array}{l}\text { Society and } \\
\text { Environment }\end{array}$ \\
\hline
\end{tabular}




\begin{tabular}{|c|c|c|c|c|c|}
\hline 6 & 1 & $\begin{array}{c}\text { Contribución de las mujeres en las revistas de } \\
\text { sociología colombianas 1959-2000 }\end{array}$ & Mayra Alejandra García Jurado & 2018 & Society \\
\hline 6 & 1 & $\begin{array}{l}\text { Consideraciones éticas en torno de la } \\
\text { documentación del Patrimonio Cultural } \\
\text { Inmaterial }\end{array}$ & Hilario Topete Lara & 2018 & Society \\
\hline 6 & 1 & $\begin{array}{l}\text { Reseña bibliográfica: Protección social y lucha } \\
\text { contra la pobreza en Brasil, Colombia y Chile. } \\
\text { ¿Graduarse de los PTC o salir de la pobreza? }\end{array}$ & Francesco María Chiodi & 2018 & Society \\
\hline 6 & 2 & $\begin{array}{l}\text { Pueblos indígenas y minorías nacionales: } \\
\text { similitudes y diferencias en la protección } \\
\text { internacional de sus derechos }\end{array}$ & María José Andrade Martínez & 2018 & Society \\
\hline 6 & 2 & $\begin{array}{l}\text { El impacto de la publicidad con equidad de } \\
\text { género en la decisión de compra de los } \\
\text { millennials }\end{array}$ & $\begin{array}{l}\text { Jovanna Nathalie Cervantes- } \\
\text { Guzmán, José G. Vargas- } \\
\text { Hernández, Guillermo Vázquez- } \\
\text { Ávila }\end{array}$ & 2018 & Society \\
\hline 6 & 2 & $\begin{array}{l}\text { Economías colaborativas, nuevas tendencias de } \\
\text { consumo y retos para Latinoamérica y Colombia }\end{array}$ & María Isabel Quintero & 2018 & Society \\
\hline 6 & 2 & $\begin{array}{c}\text { Sociología USTA: Un compromiso con la vida. } \\
\text { Balance, retos y perspectivas }\end{array}$ & $\begin{array}{l}\text { Ginneth Esmeralda Narváez } \\
\text { Jaimes }\end{array}$ & 2018 & Society \\
\hline 7 & 1 & $\begin{array}{l}\text { "Allá en La Bonga viví yo". Expresiones de } \\
\text { memoria colectiva sobre los efectos del conflicto } \\
\text { armado en Colombia }\end{array}$ & $\begin{array}{c}\text { Claudia Beltrán Romero, David } \\
\text { J. Luquetta Cediel, Clemencia } \\
\text { Rodríguez Romero, Martha } \\
\text { Romero Moreno, Luis Navarro } \\
\text { Díaz }\end{array}$ & 2019 & Society \\
\hline 7 & 1 & $\begin{array}{l}\text { Los grupos de discusión como estrategia para el } \\
\text { estudio de la subjetividad de los movimientos } \\
\text { sociales. El caso de un colectivo anarco-punk de } \\
\text { la ciudad de Aguascalientes, México }\end{array}$ & $\begin{array}{l}\text { Héctor Manuel Rodríguez } \\
\text { Figueroa }\end{array}$ & 2019 & Society \\
\hline 7 & 1 & $\begin{array}{l}\text { El caso CONPI y la crítica al movimiento } \\
\text { indígena en Colombia: aportes para repensar las } \\
\text { luchas políticas contemporáneas }\end{array}$ & $\begin{array}{l}\text { Esteban Gutiérrez Lopera, } \\
\text { Lizeth Alvarado González }\end{array}$ & 2019 & Society \\
\hline 7 & 1 & $\begin{array}{c}\text { Cohabitando hombres y espíritus, el territorio } \\
\text { embera-chamí de Cañabravita, departamento } \\
\text { del Putumayo (sur de Colombia) }\end{array}$ & Juan Carlos Rubiano Carvajal & 2019 & Society \\
\hline 7 & 1 & $\begin{array}{l}\text { Relatos de vida y transformaciones del Estado } \\
\text { en México }\end{array}$ & Raúl Eduardo Cabrera Amador & 2019 & Society \\
\hline 7 & 1 & $\begin{array}{c}\text { ¿Emprender es la solución al desempleo en } \\
\text { España? Riesgos y limitaciones }\end{array}$ & Joan Tahull Fort & 2019 & Society \\
\hline 7 & 1 & $\begin{array}{l}\text { Retratos de la guerra: glosas a propósito de } \\
\text { Susan Sontag y el fotoperiodismo de Jesús } \\
\text { Abad Colorado }\end{array}$ & $\begin{array}{l}\text { Andrés Felipe Ortiz Gordillo, } \\
\text { Giovanny Gilberto Leal } \\
\text { Roncancio }\end{array}$ & 2019 & Society \\
\hline 7 & 1 & $\begin{array}{c}\text { Aportes de la Universidad Santo Tomás a la } \\
\text { institucionalización de la Sociología en Colombia } \\
\text { en los años } 60 \text { y } 70\end{array}$ & $\begin{array}{l}\text { Guillermo Páez Morales, } \\
\text { Gregorio Clavijo Parrado, } \\
\text { Ginneth Esmeralda Narváez } \\
\text { Jaimes, Verónica Salazar } \\
\text { Baena, Miguel Urra Canales }\end{array}$ & 2019 & Society \\
\hline 7 & 1 & $\begin{array}{l}\text { "Hay que buscar medios con espacios neutros y } \\
\text { de diálogo, en donde todos podamos opinar" } \\
\text { Entrevista a Thomas Tufte, profesor de la } \\
\text { Universidad de Leicester, Reino Unido }\end{array}$ & Carlos Alberto Durán Sánchez & 2019 & Society \\
\hline 7 & 2 & $\begin{array}{l}\text { ¿Y tú qué vas a hacer con tu voto? Una mirada } \\
\text { audiovisual al plebiscito sobre el Acuerdo de Paz } \\
\text { de } 2016 \text { en Colombia }\end{array}$ & $\begin{array}{l}\text { Adolfo Baltar Moreno, Yessica } \\
\text { P. Blanco Torres, Bene del } \\
\text { Carmen Asprilla Mosquera }\end{array}$ & 2019 & Society \\
\hline 7 & 2 & $\begin{array}{c}\text { A qualitative research of humanitarian workers' } \\
\text { perceptions of preventing Gender-Based } \\
\text { Violence (GBV) in the Cox's Bazar refugee } \\
\text { camps, Bangladesh }\end{array}$ & José Navarrete & 2019 & Society \\
\hline
\end{tabular}


Identidad, memoria y arte popular: una mirada al

$\begin{array}{lll}7 & 2 & \text { centro cultural afro en el municipio de Tumaco }\end{array}$ (Colombia)

Entre la emancipación y la captura:

72 autonomización de la palabra y resistencia a las necropolíticas en los territorios indígenas nasa

(Colombia)

\section{$7 \quad 2$ El ciclo de las políticas públicas interculturales: paradojas político-antropológicas \\ $\begin{array}{lll}7 & 2 & \text { La educación rural en escenarios de paz y } \\ \text { posconflicto. Un acercamiento al estado del arte }\end{array}$ Fascismo hoy, ¿realidad concreta o treta \\ $\begin{array}{cccc}7 & 2 & \begin{array}{r}\text { Fascismo hoy, irealidad concreta o treta } \\ \text { opositora? El caso colombiano }\end{array} \\ \mathbf{7} & \mathbf{2} & \begin{array}{c}\text { Media Ecology. A Field of Study. Reflections } \\ \text { about the beginning of the Doctoral Program in } \\ \text { communication at Universidad de La Sabana }\end{array} \\ \mathbf{8} & \mathbf{1} & \begin{array}{c}\text { Referentes teóricos y metodológicos para la } \\ \text { sociología de la paz }\end{array} \\ \mathbf{8} & \mathbf{1} & \begin{array}{c}\text { Bringing Together Psychology and Peace: A } \\ \text { Critique towards the Emancipatory Potential of } \\ \text { Peace Psychology }\end{array} \\ 8 & 1 & \begin{array}{c}\text { La Paz en Práctica: caja de herramientas con } \\ \text { enfoque psicosocial para la Jurisdicción Especia }\end{array}\end{array}$ \\ $\begin{array}{cccc}7 & 2 & \begin{array}{r}\text { Fascismo hoy, irealidad concreta o treta } \\ \text { opositora? El caso colombiano }\end{array} \\ \mathbf{7} & \mathbf{2} & \begin{array}{c}\text { Media Ecology. A Field of Study. Reflections } \\ \text { about the beginning of the Doctoral Program in } \\ \text { communication at Universidad de La Sabana }\end{array} \\ \mathbf{8} & \mathbf{1} & \begin{array}{c}\text { Referentes teóricos y metodológicos para la } \\ \text { sociología de la paz }\end{array} \\ \mathbf{8} & \mathbf{1} & \begin{array}{c}\text { Bringing Together Psychology and Peace: A } \\ \text { Critique towards the Emancipatory Potential of } \\ \text { Peace Psychology }\end{array} \\ 8 & 1 & \begin{array}{c}\text { La Paz en Práctica: caja de herramientas con } \\ \text { enfoque psicosocial para la Jurisdicción Especia }\end{array}\end{array}$ \\ $\begin{array}{cccc}7 & \mathbf{2} & \begin{array}{r}\text { Fascismo hoy, irealidad concreta o treta } \\ \text { opositora? El caso colombiano }\end{array} \\ \mathbf{7} & \mathbf{2} & \begin{array}{c}\text { Media Ecology. A Field of Study. Reflections } \\ \text { about the beginning of the Doctoral Program in } \\ \text { communication at Universidad de La Sabana }\end{array} \\ \mathbf{8} & \mathbf{1} & \begin{array}{c}\text { Referentes teóricos y metodológicos para la } \\ \text { sociología de la paz }\end{array} \\ \mathbf{8} & \mathbf{1} & \begin{array}{c}\text { Bringing Together Psychology and Peace: A } \\ \text { Critique towards the Emancipatory Potential of } \\ \text { Peace Psychology }\end{array} \\ 8 & 1 & \begin{array}{c}\text { La Paz en Práctica: caja de herramientas con } \\ \text { enfoque psicosocial para la Jurisdicción Especial }\end{array}\end{array}$ \\ $\begin{array}{cccc}7 & 2 & \begin{array}{r}\text { Fascismo hoy, irealidad concreta o treta } \\ \text { opositora? El caso colombiano }\end{array} \\ \mathbf{7} & \mathbf{2} & \begin{array}{c}\text { Media Ecology. A Field of Study. Reflections } \\ \text { about the beginning of the Doctoral Program in } \\ \text { communication at Universidad de La Sabana }\end{array} \\ \mathbf{8} & \mathbf{1} & \begin{array}{c}\text { Referentes teóricos y metodológicos para la } \\ \text { sociología de la paz }\end{array} \\ \mathbf{8} & \mathbf{1} & \begin{array}{c}\text { Bringing Together Psychology and Peace: A } \\ \text { Critique towards the Emancipatory Potential of } \\ \text { Peace Psychology }\end{array} \\ 8 & 1 & \begin{array}{c}\text { La Paz en Práctica: caja de herramientas con } \\ \text { enfoque psicosocial para la Jurisdicción Especia }\end{array}\end{array}$ \\ $\begin{array}{cccc}7 & 2 & \begin{array}{r}\text { Fascismo hoy, irealidad concreta o treta } \\ \text { opositora? El caso colombiano }\end{array} \\ \mathbf{7} & \mathbf{2} & \begin{array}{c}\text { Media Ecology. A Field of Study. Reflections } \\ \text { about the beginning of the Doctoral Program in } \\ \text { communication at Universidad de La Sabana }\end{array} \\ \mathbf{8} & \mathbf{1} & \begin{array}{c}\text { Referentes teóricos y metodológicos para la } \\ \text { sociología de la paz }\end{array} \\ \mathbf{8} & \mathbf{1} & \begin{array}{c}\text { Bringing Together Psychology and Peace: A } \\ \text { Critique towards the Emancipatory Potential of } \\ \text { Peace Psychology }\end{array} \\ 8 & 1 & \begin{array}{c}\text { La Paz en Práctica: caja de herramientas con } \\ \text { enfoque psicosocial para la Jurisdicción Especia }\end{array}\end{array}$ \\ $\begin{array}{cccc}7 & 2 & \begin{array}{r}\text { Fascismo hoy, irealidad concreta o treta } \\ \text { opositora? El caso colombiano }\end{array} \\ \mathbf{7} & \mathbf{2} & \begin{array}{c}\text { Media Ecology. A Field of Study. Reflections } \\ \text { about the beginning of the Doctoral Program in } \\ \text { communication at Universidad de La Sabana }\end{array} \\ \mathbf{8} & \mathbf{1} & \begin{array}{c}\text { Referentes teóricos y metodológicos para la } \\ \text { sociología de la paz }\end{array} \\ \mathbf{8} & \mathbf{1} & \begin{array}{c}\text { Bringing Together Psychology and Peace: A } \\ \text { Critique towards the Emancipatory Potential of } \\ \text { Peace Psychology }\end{array} \\ 8 & 1 & \begin{array}{c}\text { La Paz en Práctica: caja de herramientas con } \\ \text { enfoque psicosocial para la Jurisdicción Especia }\end{array}\end{array}$ \\ $\begin{array}{cccc}7 & 2 & \begin{array}{r}\text { Fascismo hoy, irealidad concreta o treta } \\ \text { opositora? El caso colombiano }\end{array} \\ \mathbf{7} & \mathbf{2} & \begin{array}{c}\text { Media Ecology. A Field of Study. Reflections } \\ \text { about the beginning of the Doctoral Program in } \\ \text { communication at Universidad de La Sabana }\end{array} \\ \mathbf{8} & \mathbf{1} & \begin{array}{c}\text { Referentes teóricos y metodológicos para la } \\ \text { sociología de la paz }\end{array} \\ \mathbf{8} & \mathbf{1} & \begin{array}{c}\text { Bringing Together Psychology and Peace: A } \\ \text { Critique towards the Emancipatory Potential of } \\ \text { Peace Psychology }\end{array} \\ 8 & 1 & \begin{array}{c}\text { La Paz en Práctica: caja de herramientas con } \\ \text { enfoque psicosocial para la Jurisdicción Especia }\end{array}\end{array}$ \\ $\begin{array}{ccc}7 & \mathbf{2} & \begin{array}{r}\text { Fascismo hoy, irealidad concreta o treta } \\ \text { opositora? El caso colombiano }\end{array} \\ \mathbf{7} & \mathbf{2} & \begin{array}{c}\text { Media Ecology. A Field of Study. Reflections } \\ \text { about the beginning of the Doctoral Program in } \\ \text { communication at Universidad de La Sabana }\end{array} \\ \mathbf{8} & \mathbf{1} & \begin{array}{c}\text { Referentes teóricos y metodológicos para la } \\ \text { sociología de la paz }\end{array} \\ \mathbf{8} & \mathbf{1} & \begin{array}{c}\text { Bringing Together Psychology and Peace: A } \\ \text { Critique towards the Emancipatory Potential of } \\ \text { Peace Psychology }\end{array} \\ 8 & 1 & \begin{array}{c}\text { La Paz en Práctica: caja de herramientas con } \\ \text { enfoque psicosocial para la Jurisdicción Especia }\end{array}\end{array}$ \\ $\begin{array}{cccc}7 & 2 & \begin{array}{r}\text { Fascismo hoy, irealidad concreta o treta } \\ \text { opositora? El caso colombiano }\end{array} \\ \mathbf{7} & \mathbf{2} & \begin{array}{c}\text { Media Ecology. A Field of Study. Reflections } \\ \text { about the beginning of the Doctoral Program in } \\ \text { communication at Universidad de La Sabana }\end{array} \\ \mathbf{8} & \mathbf{1} & \begin{array}{c}\text { Referentes teóricos y metodológicos para la } \\ \text { sociología de la paz }\end{array} \\ \mathbf{8} & \mathbf{1} & \begin{array}{c}\text { Bringing Together Psychology and Peace: A } \\ \text { Critique towards the Emancipatory Potential of } \\ \text { Peace Psychology }\end{array} \\ 8 & 1 & \begin{array}{c}\text { La Paz en Práctica: caja de herramientas con } \\ \text { enfoque psicosocial para la Jurisdicción Especia }\end{array}\end{array}$ \\ $\begin{array}{cccc}7 & 2 & \begin{array}{r}\text { Fascismo hoy, irealidad concreta o treta } \\ \text { opositora? El caso colombiano }\end{array} \\ \mathbf{7} & \mathbf{2} & \begin{array}{c}\text { Media Ecology. A Field of Study. Reflections } \\ \text { about the beginning of the Doctoral Program in } \\ \text { communication at Universidad de La Sabana }\end{array} \\ \mathbf{8} & \mathbf{1} & \begin{array}{c}\text { Referentes teóricos y metodológicos para la } \\ \text { sociología de la paz }\end{array} \\ \mathbf{8} & \mathbf{1} & \begin{array}{c}\text { Bringing Together Psychology and Peace: A } \\ \text { Critique towards the Emancipatory Potential of } \\ \text { Peace Psychology }\end{array} \\ 8 & 1 & \begin{array}{c}\text { La Paz en Práctica: caja de herramientas con } \\ \text { enfoque psicosocial para la Jurisdicción Especia }\end{array}\end{array}$ \\ $\begin{array}{ccc}7 & \mathbf{2} & \begin{array}{c}\text { Fascismo hoy, irealidad concreta o treta } \\ \text { opositora? El caso colombiano }\end{array} \\ \mathbf{7} & \mathbf{2} & \begin{array}{c}\text { Media Ecology. A Field of Study. Reflections } \\ \text { about the beginning of the Doctoral Program in } \\ \text { communication at Universidad de La Sabana }\end{array} \\ \mathbf{8} & \mathbf{1} & \begin{array}{c}\text { Referentes teóricos y metodológicos para la } \\ \text { sociología de la paz }\end{array} \\ \mathbf{8} & \mathbf{1} & \begin{array}{c}\text { Bringing Together Psychology and Peace: A } \\ \text { Critique towards the Emancipatory Potential of } \\ \text { Peace Psychology }\end{array} \\ 8 & 1 & \begin{array}{c}\text { La Paz en Práctica: caja de herramientas con } \\ \text { enfoque psicosocial para la Jurisdicción Especial }\end{array}\end{array}$} para la Paz

\begin{tabular}{|c|c|c|c|c|c|}
\hline 8 & 1 & $\begin{array}{l}\text { La metáfora de la corporalidad en la guerra } \\
\text { como dispositivo resiliente }\end{array}$ & $\begin{array}{l}\text { Angélica María Villamil } \\
\text { Benavides, Adrián David } \\
\text { Galindo Ubaque }\end{array}$ & 2020 & Society \\
\hline 8 & 1 & $\begin{array}{l}\text { Democracia participativa y planeación del } \\
\text { desarrollo en los programas de desarrollo con } \\
\text { enfoque territorial }\end{array}$ & $\begin{array}{l}\text { Germán Darío Valencia } \\
\text { Agudelo, Daniel Restrepo-Cano }\end{array}$ & 2020 & Society \\
\hline 8 & 1 & $\begin{array}{l}\text { Buscando los orígenes en El día señalado: la } \\
\text { agricultura como respuesta a la violencia }\end{array}$ & Jaime A. Orrego & 2020 & $\begin{array}{l}\text { Society and } \\
\text { Environment }\end{array}$ \\
\hline 8 & 1 & $\begin{array}{l}\text { La construcción de paz a través de la } \\
\text { consolidación del conocimiento biológico y } \\
\text { territorial }\end{array}$ & $\begin{array}{c}\text { Jair Hernando Castro Romero, } \\
\text { Edwin Alexander Sanabria } \\
\text { Ospina }\end{array}$ & 2020 & $\begin{array}{l}\text { Society and } \\
\text { Environment }\end{array}$ \\
\hline 8 & 1 & $\begin{array}{c}\text { Factores sociales, económicos y ambientales de } \\
\text { las organizaciones de economía solidaria } \\
\text { conformadas en situaciones de crisis en } \\
\text { Colombia }\end{array}$ & Ana Milena Silva Valencia & 2020 & $\begin{array}{l}\text { Society and } \\
\text { Environment }\end{array}$ \\
\hline 8 & 1 & $\begin{array}{l}\text { Literatura y guerra. Elementos de una poética de } \\
\text { la escucha en la obra Desterrados. Crónicas del } \\
\text { desarraigo, de Alfredo Molano }\end{array}$ & Michelle Camila Pérez Cardozo & 2020 & Society \\
\hline 8 & 1 & $\begin{array}{l}\text { Lectura, escritura y oralidad: la narración } \\
\text { colectiva de Colombia en tiempos de } \\
\text { posconflicto }\end{array}$ & $\begin{array}{c}\text { Natalia Montejo Vélez, Federico } \\
\text { López }\end{array}$ & 2020 & Society \\
\hline 8 & 1 & $\begin{array}{l}\text { Quintín Lame, conciencia histórica y decreto } \\
\text { autonómico. Rutas para la paz y la vida en } \\
\text { Colombia }\end{array}$ & $\begin{array}{l}\text { Víctor Alonso Molina Bedoya, } \\
\text { Arnulfo Hurtado Cerón }\end{array}$ & 2020 & Society \\
\hline 8 & 1 & $\begin{array}{c}\text { Configuración de creencias sociales y } \\
\text { orientaciones emocionales colectivas en } \\
\text { ciudadanos de Sonsón y Cocorná (Antioquia) } \\
\text { sobre el conflicto armado, el proceso de paz y la } \\
\text { reconciliación }\end{array}$ & $\begin{array}{l}\text { Juan David Villa Gómez, María } \\
\text { Camila Agudelo López, Susana } \\
\text { Hoyos, Valentina Castro, } \\
\text { Cristian Evelio Buitrago, Natali } \\
\text { Velásquez Cuartas }\end{array}$ & 2020 & Society \\
\hline 8 & 1 & $\begin{array}{l}\text { La experiencia psicológica del agresor en el } \\
\text { conflicto violento }\end{array}$ & $\begin{array}{l}\text { María Prieto-Ursúa, Ángela } \\
\text { Ordóñez Carabaño }\end{array}$ & 2020 & Society \\
\hline 8 & 1 & $\begin{array}{l}\text { Construir la paz en Colombia: implicaciones del } \\
\text { restablecimiento de la dignidad humana }\end{array}$ & $\begin{array}{l}\text { Laura Alejandra Uribe Gonima, } \\
\text { Laura Camila Velasco Vélez }\end{array}$ & 2020 & Society \\
\hline
\end{tabular}

$\begin{array}{llr}\text { Alfonso Barquín Cendejas } & 2019 & \text { Society } \\ \text { Leidy Johana Trujillo Franco, } & 2019 \quad \text { Society and }\end{array}$ Silvio Cardona González $\quad 2019$ Environment

Luis Carlos Castro Riaño 2019 Society

Daniel E. Aguilar Rodríguez 2019 Environment

Ginneth Narvaez, Camilo

Castiblanco, Miguel Urra Canales

Mónica Catarina Pereira

Soares, Ana Margarida Sá $2020 \quad$ Society Caetano, Mariana Reis Barbosa

Paula Andrea Ruiz Álvarez,

Sergio Daniel Arrieta Vera, Aida

Milo Daniel Arieta Vera, Aida

2020 Society

Patricia Vaca Vaca

navides, Adrián David

2020 Society

Society

La metáfora de la corporalidad en la guerra como dispositivo resiliente

Germán Darío Valencia

Agudelo, Daniel Restrepo-Cano

Luisa Fernanda Larios Beltrán $2019 \quad$ Society

Simone Ferrari $2019 \begin{gathered}\begin{array}{c}\text { Society and } \\ \text { Environment }\end{array} \\ \text { Eniri }\end{gathered}$

Society and
Environment 


\begin{tabular}{|c|c|c|c|c|c|}
\hline 8 & 1 & $\begin{array}{l}\text { Actores civiles no estatales en el mantenimiento } \\
\text { y la consolidación de la paz en África Occidental }\end{array}$ & $\begin{array}{l}\text { Abu Bakarr Bah, Nastassja } \\
\text { Rojas Silva }\end{array}$ & 2020 & Society \\
\hline 8 & 1 & $\begin{array}{c}\text { La resolución de conflictos: ancestros } \\
\text { tradicionales africanos, parentesco y rituales de } \\
\text { reconciliación }\end{array}$ & $\begin{array}{l}\text { Kathryn Coe, Craig T. Palmer, } \\
\text { Khadijah, Andrea Leiva Espitia }\end{array}$ & 2020 & Society \\
\hline 8 & 1 & $\begin{array}{c}\text { Reseña del libro 'Guerrilla marketing. } \\
\text { Contrainsurgencia y capitalismo en Colombia', } \\
\text { de Alexander L. Fattal }\end{array}$ & Piedad Gómez Castillo & 2020 & Society \\
\hline 8 & 1 & $\begin{array}{c}\text { Reseña del libro 'Reconciliaciones y resistencias. } \\
\text { Modelos mentales y aprendizajes colectivos en } \\
\text { la construcción de paz territorial en Colombia', } \\
\text { de Jhon Alexánder Idrobo Velasco y Johanna } \\
\text { Amaya Panche }\end{array}$ & Diego Alejandro Álvarez Pinzón & 2020 & Society \\
\hline
\end{tabular}

From this table, it was possible to identify that the most constant field in the articles published by this magazine is Society, with a presence in 84 articles, representing $82.4 \%$ of its total publications; In the case of Environment, although within the studies of social sciences in recent years it has become a recurring theme within it, in the calls made by the previous editors of this magazine, no articles have been received and published mostly that develop the environmental theme as a central feature, since there is only record in 9 articles, which represents $8.8 \%$ of the magazine. Finally, and as a finding of the publications, it was possible to register that from the second number published by this magazine for 2013 , there begins to be a greater presence of texts that work together to mix the fields of Environment and Society, with a total of 9 articles and $8.8 \%$ of the total presence.

Table 2: Field consolidation.

\begin{tabular}{|c|c|c|}
\hline FIELD & Articles & $\%$ \\
\hline Society & 84 & $82.4 \%$ \\
\hline Environment & 9 & $8.8 \%$ \\
\hline $\begin{array}{c}\text { Society and } \\
\text { environment }\end{array}$ & 9 & $8.8 \%$ \\
\hline
\end{tabular}

Subsequently, and to establish a less speculative analysis process, the contents of each of the articles were evaluated in relation to the fields and subfields mentioned above, in order to construct classifying tables that numerically reflect the presence score of each subfield in the articles. totals for the entire journal, in order to consolidate large academic work groups in the research processes and publication of the journal texts. 
Table 3: Analysis of the "Society" field

\begin{tabular}{|c|c|c|}
\hline Subfield & $\begin{array}{l}\text { Number of } \\
\text { articles } \\
\text { covered }\end{array}$ & $\begin{array}{c}\% \text { of journal articles in which } \\
\text { this field is discussed }(\mathrm{N}= \\
102)\end{array}$ \\
\hline Equality and justice & 79 & $77 \%$ \\
\hline Communication & 74 & $73 \%$ \\
\hline Peace and coexistence & 69 & $68 \%$ \\
\hline Citizen participation & 58 & $57 \%$ \\
\hline Social systems & 47 & $46 \%$ \\
\hline Economic and social development & 39 & $38 \%$ \\
\hline Knowledge management & 32 & $31 \%$ \\
\hline Social groups & 32 & $31 \%$ \\
\hline Education & 28 & $27 \%$ \\
\hline Human rights & 26 & $25 \%$ \\
\hline Public politics & 23 & $23 \%$ \\
\hline Infrastructure & 20 & $20 \%$ \\
\hline Town planning & 20 & $20 \%$ \\
\hline Social behavior & 18 & $18 \%$ \\
\hline Information literacy & 19 & $19 \%$ \\
\hline Economy & 15 & $15 \%$ \\
\hline Information users & 14 & $14 \%$ \\
\hline $\begin{array}{l}\text { Health, Well-being and public } \\
\text { health }\end{array}$ & 13 & $13 \%$ \\
\hline Value systems & 8 & $8 \%$ \\
\hline $\begin{array}{l}\text { Scientific developments applied to } \\
\text { the transformation of society }\end{array}$ & 5 & $5 \%$ \\
\hline
\end{tabular}

As can be seen, more than half of the articles published focus on "Equality and justice", "Communication", "Peace and coexistence" and "Citizen participation". On the opposite side, 
the least worked subfields in the articles have been "Economy", "Information users", "Health, well-being and public health", "Value systems" and "Scientific developments applied to the transformation of society". Faced with these values, it is important to highlight that the magazine's promoting faculties (Social Communication, Sociology and Graphic Design) have their emphasis on the most published topics.

\section{ENVIRONMENT}

Table 4: Analysis of the "Society" field

\begin{tabular}{|c|c|c|}
\hline Subfield & $\begin{array}{l}\text { Number of } \\
\text { articles covered }\end{array}$ & $\begin{array}{l}\% \text { of journal articles in which this } \\
\text { field is discussed }(N=102)\end{array}$ \\
\hline Environments & 10 & $10 \%$ \\
\hline Effects on human activities & 7 & $7 \%$ \\
\hline $\begin{array}{l}\text { Environmental } \\
\text { management }\end{array}$ & 6 & $6 \%$ \\
\hline Habitat & 5 & $5 \%$ \\
\hline Ecology & 5 & $5 \%$ \\
\hline Sustainable cities & 5 & $5 \%$ \\
\hline $\begin{array}{l}\text { Responsible production and } \\
\text { consumption }\end{array}$ & 4 & $4 \%$ \\
\hline Biodiversity & 3 & $3 \%$ \\
\hline Pollution & 2 & $2 \%$ \\
\hline $\begin{array}{l}\text { Clean and sustainable } \\
\text { energy }\end{array}$ & 2 & $2 \%$ \\
\hline Smart cities & 1 & $1 \%$ \\
\hline Atmosphere & 0 & $0 \%$ \\
\hline Aquatic ecosystems & 0 & $0 \%$ \\
\hline Terrestrial ecosystems & 0 & $0 \%$ \\
\hline
\end{tabular}

As previously noted, the presence of the environmental subfields is still incipient in the magazine. This may be due to the fact that, in the academic world, "we can identify a progressive approach from" the environmental "to" the social ". However, perhaps the approach of "the social" towards "the environmental" is still a pending subject (Urra, M. 2014). 


\section{CONCLUSIONS}

It is far from the intention of this article to identify the success or the mistake of the editorial line of the magazine. Rather, it is about offering a baseline on which to reflect on the alignment of academic journals with educational projects from which it is promoted and on which, in a modest way, to make some recommendation in the future so that this alignment is strengthened.

In this sense, it seems that a magazine promoted by a Division of Social Sciences, has focused on the field of action "Society", which is the one with the highest disciplinary affinity. In addition, the most worked subfields are in accordance with the emphasis of the Division's programs. This does not mean that, in the future, greater weight is desirable, not so much for pure environmental issues, but for articles that bring the "Society" field into dialogue with the "Environment" field. For this, the editorial team of the magazine would be recommended to promote monographic issues, where specific calls can be opened to promote the reception of texts that combine both fields.

Finally, it is pertinent to highlight the importance of this type of research on the approaches of academic journals. In a context where it is published more and more quickly, it is necessary to make a "stop along the way" and rigorously analyze how the editorial line proposals are being developed and how they are finally materialized in the approach or not of certain topics.

\section{Bibliography}

Agudelo, G. D. V., \& Restrepo-Cano, D. (2020). Democracia participativa y planeación del desarrollo en los programas de desarrollo con enfoque territorial. Campos en Ciencias Sociales, 8(1), Article 1. https://doi.org/10.15332/25006681/5260

Albarracín, F. L. R. (2014). Bibliographic review: Emergencies of the territory and local communication. Experiences of communication and development about the environment in Colombia. Campos en Ciencias Sociales, 2(2), 315-318.

Álvarez, P. A. R., Vera, S. D. A., Lozano, A. M. C., \& Vaca, M. P. V. (2020). La Paz en Práctica: Caja de herramientas con enfoque psicosocial para la Jurisdicción Especial para la Paz. Campos en Ciencias Sociales, 8(1), Article 1. https://doi.org/10.15332/25006681/5716

Amador, R. E. C. (2019). Relatos de vida y transformaciones del Estado en México. Campos en Ciencias Sociales, 7(1), 127-159. https://doi.org/10.15332/25006681.4687

Andrade, G. A. (2015). Presentación del número. Campos en Ciencias Sociales, 3(1), 9-10.

Andrade, G. A. (2017). Editorial. Campos en Ciencias Sociales, 5(1 y 2), 9-10.

Andrade, G. A. (2018a). Presentación del número. Campos En Ciencias Sociales, 6(1), 910.

Andrade, G. A. (2018b). Presentación del número. Campos en Ciencias Sociales, 6(2), 910.

Andrade, G. A. (2019). Presentación del número. Campos en Ciencias Sociales, 7(1), 9-16.

Andrade, G. A., \& Hincapié, J. I. J. (2015). Editorial. Campos en Ciencias Sociales, 3(2), 125-128.

Andrade, G. K. A. (2019). Presentación. Campos en Ciencias Sociales, 7(2), 9-15.

Assis, J. M. F. (2014). Archivo, memoria y dictadura: Activistas religiosos y enfrentamiento al régimen dictatorial en Brasill. Campos en Ciencias Sociales, 2(1), 51-79. https://doi.org/10.15332/s2339-3688.2014.0001.01

Ayure, M., \& Andrade, G. A. (2016a). Editorial. Campos en Ciencias Sociales, 4(1), 7-7.

Ayure, M., \& Andrade, G. A. (2016b). Presentación del número. Campos en Ciencias Sociales, 4(2), 145-146. 
Bah, A. B., \& Silva, N. R. (2020). Actores civiles no estatales en el mantenimiento y la consolidación de la paz en África Occidental. Campos en Ciencias Sociales, 8(1), Article 1. https://revistas.usantotomas.edu.co/index.php/campos/article/view/5724

Barrios, M. G. M. (2016). Prácticas sociales, creatividad y habitar en las regiones Medellín, Antioquia (Colombia). Campos en Ciencias Sociales, 4(2), 219-233. http://dx.doi.org/10.15332/s2339-3688.2016.0002.04

Bedoya, V. A. M., \& Cerón, A. H. (2020). Quintín Lame, conciencia histórica y decreto autonómico. Rutas para la paz y la vida en Colombia. Campos en Ciencias Sociales, 8(1), Article 1. https://doi.org/10.15332/25006681/5074

Bello, L. A. S., \& Pineda, M. P. (2013). La construcción discursiva de un genocidio en Colombia: Una aproximación a la versión de las Fuerzas Militares en el caso de la Unión Patriótica. Campos en Ciencias Sociales, 1(2), 279-298. https://doi.org/10.15332/s2339-3688.2013.0002.03

Beltrán, L. F. L. (2019). Identidad, memoria y arte popular: Una mirada al centro cultural afro en el municipio de Tumaco (Colombia). Campos en Ciencias Sociales, 7(2), 75124. https://doi.org/10.15332/25006681.50061

Benavides, A. M. V., \& Ubaque, A. D. G. (2020). La metáfora de la corporalidad en la guerra como dispositivo resiliente. Campos en Ciencias Sociales, 8(1), Article 1. https://doi.org/10.15332/25006681/5717

Bernal, A. C. (2014). La Hacienda El Carmen como escenario coyuntural de disputa sobre memoria, territorio y patrimonio. Campos en Ciencias Sociales, 2(2), 179-205. https://doi.org/10.15332/s2339-3688.2014.0002.01

Berti, S., \& Cantú, A. (2013). Protestar por los derechos, el derecho a protestar. Una lectura desde las audiencias. Campos en Ciencias Sociales, 1(1), 125-148.

Canales, M. U., \& Muñoz, L. C. P. (2020). Editorial. Campos en Ciencias Sociales, 8(1), Article 1. https://revistas.usantotomas.edu.co/index.php/campos/article/view/5751

Canoas, M. C. (2015). La planeación en el municipio y resguardo de Jambaló (Colombia). Campos en Ciencias Sociales, 3(2), 239-260. https://doi.org/10.15332/s23393688.2015.0002.06

Cardozo, M. C. P. (2020). Literatura y guerra. Elementos de una poética de la escucha en la obra Desterrados. Crónicas del desarraigo, de Alfredo Molano. Campos en Ciencias Sociales, 8(1), Article 1. https://doi.org/10.15332/25006681/5720

Carvajal, J. C. R. (2019). Cohabitando hombres y espíritus, el territorio embera-chamí de Cañabravita, departamento del Putumayo (sur de Colombia). Campos en Ciencias Sociales, 7(1), 103-125. https://doi.org/10.15332/25006681.4758

Castillo, P. G. (2020). Reseña del libro «Guerrilla marketing. Contrainsurgencia y capitalismo en Colombia», de Alexander L. Fattal. Campos en Ciencias Sociales, 8(1), Article 1. https://revistas.usantotomas.edu.co/index.php/campos/article/view/5726

Cendejas, A. B. (2019). El ciclo de las políticas públicas interculturales: Paradojas políticoantropológicas. Campos en Ciencias Sociales, 7(2), 147-174. https://doi.org/10.15332/25006681.5105

Cervantes-Guzmán, J. N., Vargas-Hernández, J. G., \& Vázquez-Ávila, G. (2018). El impacto de la publicidad con equidad de género en la decisión de compra de los millennials. Campos En Ciencias Sociales, 6(2), 49-93. https://doi.org/10.15332/s23393688.2018.0002.02

Chiodi, F. M. (2018). Reseña bibliográfica: Protección social y lucha contra la pobreza en Brasil, Colombia y Chile. ¿Graduarse de los PTC o salir de la pobreza? Campos en Ciencias Sociales, 6(1), 115-122. https://doi.org/10.15332/s2339-3688.2018.0001.05

Coe, K., Palmer, C. T., elShabazz, K., \& Espitia, A. L. (2020). La resolución de conflictos: Ancestros tradicionales africanos, parentesco y rituales de reconciliación. Campos en Ciencias Sociales, 8(1), Article 1. https://revistas.usantotomas.edu.co/index.php/campos/article/view/5725

Cruz, M. P. R. (2017). Un acercamiento al patrimonio cultural inmaterial su salvaguarda y patrimonialización. Campos en Ciencias Sociales, 5(1 y 2), 175-209. 
Delgado, M. C. S. (2018). Redes que se construyen dentro de la comunidad indígena Embera desplazada en Bogotá (Colombia): Caso del alojamiento Embera en Santafé. Campos en Ciencias Sociales, 6(1), 13-41. https://doi.org/10.15332/s23393688.2018.0001.01

Durán, C. A. C. (2013). Presentación: Campos de conocimiento, investigación y transformación en ciencias sociales. Campos en Ciencias Sociales, 1(1), 11-15.

Ferrari, S. (2019). Entre la emancipación y la captura: Autonomización de la palabra y resistencia a las necropolíticas en los territorios indígenas nasa (Colombia). Campos en Ciencias Sociales, 7(2), 125-146. https://doi.org/10.15332/25006681.5019

Figueroa, H. M. R. (2019). Los grupos de discusión como estrategia para el estudio de la subjetividad de los movimientos sociales. El caso de un colectivo anarco-punk de la ciudad de Aguascalientes, México. Campos en Ciencias Sociales, 7(1), 43-76. https://doi.org/10.15332/25006681.4697

Fort, J. T. (2019). ¿Emprender es la solución al desempleo en España? Riesgos y limitaciones. Campos en Ciencias Sociales, 7(1), 161-193. https://doi.org/10.15332/25006681.4543

Franco, L. J. T., \& González, S. C. (2019). La educación rural en escenarios de paz y posconflicto. Un acercamiento al estado del arte. Campos en Ciencias Sociales, 7(2), 175-218. https://doi.org/10.15332/25006681.4288

Gavilán, C. G. (2013). Hermenéutica de los cuerpos. Campos en Ciencias Sociales, 1(2), 313-333. https://doi.org/10.15332/s2339-3688.2013.0002.05

Gélvez, M. V. R., Huérfano, E. D. R. H., \& Sandoval, C. A. M. (2013). A la zaga de una propuesta para la combinación de metodologías de investigación. Campos en Ciencias Sociales, 1(2), 335-362. https://doi.org/10.15332/s2339-3688.2013.0002.06

Gil, M. E. G. (2013). El uso de la imagen como herramienta de investigación. Campos en Ciencias Sociales, 1(2), 363-372. https://doi.org/10.15332/s2339-3688.2013.0002.07

Giraldo, M. A. A. (2017). Periodismo regional y conflictos sociales. Análisis de las opiniones de los diarios Correo y Los Andes de Puno (Perú) durante el conflicto social del "Aimarazo" (2011). Campos en Ciencias Sociales, 5(1 y 2), 13-37. https://doi.org/10.15332/s2339-3688.2017.0001.01

Gómez, J. D. V., López, M. C. A., Hoyos, S., Castro, V., Buitrago, C. E., \& Cuartas, N. V. (2020). Configuración de creencias sociales y orientaciones emocionales colectivas en ciudadanos de Sonsón y Cocorná (Antioquia) sobre el conflicto armado, el proceso de paz y la reconciliación. Campos en Ciencias Sociales, 8(1), Article 1. https://doi.org/10.15332/25006681/5274

Gómez, M. M. B. (2013). Las organizaciones comunitarias como instituciones intermedias: Las Juntas de Acción Comunal en el municipio de Tenjo. Campos en Ciencias Sociales, 1(1), 17-41.

Gómez, R. (2017). Aproximación a las prácticas ciudadanas en la escuela: El caso del colegio Fernando González Ochoa de la localidad de Usme (Bogotá, Colombia). Campos en Ciencias Sociales, 5(1 y 2), 115-135. https://doi.org/10.15332/s23393688.2017.0001.04

Gómez-Montañez, P. F. (2014). Sacralidad, tormento y rescate: La vida social de la chicha muisca. Campos en Ciencias Sociales, 2(1), 139-160. https://doi.org/10.15332/s2339$\underline{3688.2014 .0001 .04}$

Gonima, L. A. U., \& Vélez, L. C. V. (2020). Construir la paz en Colombia: Implicaciones del restablecimiento de la dignidad humana. Campos en Ciencias Sociales, 8(1), Article 1. https://doi.org/10.15332/25006681/5723

González, M. T. S. (2015). Aproximación al estudio de las formas de representación de los actores armados en justicia y paz. Campos en Ciencias Sociales, 3(2), 153-176. https://doi.org/10.15332/s2339-3688.2015.0002.02

Gordillo, A. F. O., \& Roncancio, G. G. L. (2019). Retratos de la guerra: Glosas a propósito de Susan Sontag y el fotoperiodismo de Jesús Abad Colorado. Campos en Ciencias Sociales, 7(1), 195-225. https://doi.org/10.15332/25006681.4690 
Houtart, F. (2015). La dinámica social de las orientaciones de desarrollo en América Latina. Campos en Ciencias Sociales, 3(1), 97-110. https://doi.org/10.15332/s23393688.2015.0001.05

Jaimes, Ginneth Esmeralda Narváez. (2018). Sociología USTA: Un compromiso con la vida. Balance, retos y perspectivas. Campos En Ciencias Sociales, 6(2), 119-127. https://doi.org/10.15332/s2339-3688.2018.0002.04

Jaimes, Ginneth Esmeralda Narvaez, Castiblanco, C., \& Canales, M. U. (2020). Referentes teóricos y metodológicos para la sociología de la paz. Campos en Ciencias Sociales, 8(1), Article 1. https://doi.org/10.15332/25006681/5453

Jurado, M. A. G. (2018). Contribución de las mujeres en las revistas de sociología colombianas 1959-2000. Campos en Ciencias Sociales, 6(1), 73-90. https://doi.org/10.15332/s2339-3688.2018.0001.03

Lara, H. T. (2018). Consideraciones éticas en torno de la documentación del Patrimonio Cultural Inmaterial. Campos En Ciencias Sociales, 6(1), 91-114. https://doi.org/10.15332/s2339-3688.2018.0001.04

Lopera, E. G., \& González, L. A. (2019). El caso CONPI y la crítica al movimiento indígena en Colombia: Aportes para repensar las luchas políticas contemporáneas. Campos en Ciencias Sociales, 7(1), 77-101. https://doi.org/10.15332/25006681.4998

López, L. R. T. (2013). In memoriam: La plataforma massmediática como dispositivo de emancipación. Campos en Ciencias Sociales, 1(1), 197-209.

Manrique, A. C. S. (2014). Una mirada a la cátedra de estudios afrocolombianos a partir de las reflexiones de los líderes de la Red Elegguá en la ciudad de Bogotá. Campos en Ciencias Sociales, 2(2), 225-256. https://doi.org/10.15332/s2339-3688.2014.0002.03

Marín, C. A. M. (2014). En busca de la alteridad. Campos en Ciencias Sociales, 2(2), 257 285. https://doi.org/10.15332/s2339-3688.2014.0002.05

Marín, J. G. A. (2014a). Editorial. Campos en Ciencias Sociales, 2(1), 7-8.

Marín, J. G. A. (2014b). Editorial. Campos en Ciencias Sociales, 2(2), 175-176.

Martínez, M. J. A. (2018). Pueblos indígenas y minorías nacionales: Similitudes y diferencias en la protección internacional de sus derechos. Campos En Ciencias Sociales, 6(2), 13-48. https://doi.org/10.15332/s2339-3688.2018.0002.01

Mengo, R. I., \& Tenaglia, P. R. (2015). La narrativa como herramienta didáctica y de comunicación para la enseñanza de la historia social contemporánea y reciente. Campos en Ciencias Sociales, 3(1), 35-50. https://doi.org/10.15332/s23393688.2015 .0001 .02

Mojica, S. J. (2013). Memoria, prácticas artísticas y espacio público: Posibilidades frente al conflicto armado colombiano. Campos en Ciencias Sociales, 1(2), 387-413. https://doi.org/10.15332/s2339-3688.2013.0002.09

Montoya, A. N. (2013). Mercado y consumo: Economía política de las telecomunicaciones en Colombia. Campos en Ciencias Sociales, 1(2), 231-250. https://doi.org/10.15332/s2339-3688.2013.0002.01

Mora, E. G. (2015). A propósito de los "falsos opuestos" en Ciencias Sociales -La IAP como posibilidad para la lectura integral y crítica de la realidad-. Campos en Ciencias Sociales, 3(2), 261-279. https://doi.org/10.15332/s2339-3688.2015.0002.07

Morales, G. P., Parrado, G. C., Jaimes, G. E. N., Baena, V. S., \& Canales, M. U. (2019). Aportes de la Universidad Santo Tomás a la institucionalización de la Sociología en Colombia en los años 60 y 70. Campos En Ciencias Sociales, 7(1), 227-247. https://doi.org/10.15332/25006681.4549

Moreno, A. B., Torres, Y. P. B., \& Mosquera, B. del C. A. (2019). ¿Y tú qué vas a hacer con tu voto? Una mirada audiovisual al plebiscito sobre el Acuerdo de Paz de 2016 en Colombia. Campos en Ciencias Sociales, 7(2), 17-44. https://doi.org/10.15332/25006681.5030

Muñoz, C. A. R. (2017). Voces testimoniales y realidad experimental en Guadalupe años sin cuenta del grupo la Candelaria. Campos en Ciencias Sociales, 5(1 y 2), 159-174.

Murillo, A. M. V. (2016). La memoria como eje de cambio social en la escuela. Campos en Ciencias Sociales, 4(2), 149-165. http://dx.doi.org/10.15332/s2339-3688.2016.0002.01 
Navarrete, J. (2019). A qualitative research of humanitarian workers' perceptions of preventing Gender-Based Violence (GBV) in the Cox's Bazar refugee camps, Bangladesh. Campos En Ciencias Sociales, 7(2), 45-74. https://doi.org/10.15332/25006681.4670

Nieto, L. P. M. (2015). Participación de los padres de familia en la escuela. La violencia como mediadora de la relación entre los padres y la escuela. Campos en Ciencias Sociales, 3(2), 215-237. https://doi.org/10.15332/s2339-3688.2015.0002.05

Orrego, J. A. (2020). Buscando los orígenes en El día señalado: La agricultura como respuesta a la violencia. Campos en Ciencias Sociales, 8(1), Article 1. https://doi.org/10.15332/25006681/5718

Ostos-Ortiz, O. L., \& Cortés-Gallego, M. A. (s. f.). Los campos de acción en la Universidad Santo Tomás. Resultados del estudio 2012-2018. Revista interamericana de investigación, educación y pedagogía, 12(1), 15-40.

Padre Jorge Ferdinando Rodríguez Ruiz, O. P. (2013). From the Director. Campos en Ciencias Sociales, 1(2), 221-229.

Parada, C. C., Mendoza, C. U., \& Sáez, F. A. (2016). Los imaginarios sociales desde Armando Silva, sus avances, transformaciones y productos. Campos en Ciencias Sociales, 4(1), 81-100.

Perdomo, O. L. (2013). Sobre mujeres y desplazamiento. Campos en Ciencias Sociales, 1(1), 149-168. http://dx.doi.org/10.15332/s2339-3688.2016.0001.04

Pérez, G. T., \& Rodríguez, D. E. A. (2015). Comportamiento humano y prevención de dengue: Estudio en barrios endémicos de Barranquilla, Bucaramanga y Armenia (Colombia). Campos en Ciencias Sociales, 3(2), 199-214. https://doi.org/10.15332/s2339-3688.2015.0002.04

Pinzón, D. A. Á. (2020). Reseña del libro «Reconciliaciones y resistencias. Modelos mentales y aprendizajes colectivos en la construcción de paz territorial en Colombia», de Jhon Alexánder Idrobo Velasco y Johanna Amaya Panche. Campos en Ciencias Sociales, 8(1), Article 1. https://revistas.usantotomas.edu.co/index.php/campos/article/view/5727

Prieto-Ursúa, M., \& Carabaño, Á. O. (2020). La experiencia psicológica del agresor en el conflicto violento. Campos en Ciencias Sociales, 8(1), Article 1. https://doi.org/10.15332/25006681/5722

Quintero, M. I. (2018). Economías colaborativas, nuevas tendencias de consumo y retos para Latinoamérica y Colombia. Campos En Ciencias Sociales, 6(2), 95-118. https://doi.org/10.15332/s2339-3688.2018.0002.03

Quiñones, A. H. (2013). Patrimonio cultural y turismo en San Pedro de Atacama, Chile. Campos en Ciencias Sociales, 1(2), 299-311. https://doi.org/10.15332/s23393688.2013.0002.04

Riaño, L. C. C. (2019). Fascismo hoy, ¿realidad concreta o treta opositora? El caso colombiano. Campos en Ciencias Sociales, 7(2), 219-236. https://doi.org/10.15332/25006681.4970

Ribeiro, J. F. (2014). Historia, memoria y olvido: La historia contada sobre el levantamiento de 1935 en el Partido Comunista de Brasil (1943-1958). Campos en Ciencias Sociales, 2(1), 81-109. https://doi.org/10.15332/s2339-3688.2014.0001.02

Rincón, A. A. A. (2016). La comunicación visual a través de Pepe Mexía y Ricardo Rendón, 1915-1930. Campos en Ciencias Sociales, 4(1), 33-57. https://doi.org/10.15332/s2339-3688.2016.0001.02

Rincón, L. A. (2013). Modelo de televisión comunitaria para el desarrollo humano: Estudio de la televisión comunitaria de Colombia y nueva propuesta de programación para su audiencia. Campos en Ciencias Sociales, 1(1), 107-123.

Rincón, L. A. (2016). Comunicación y deporte: Un campo integrador para el análisis del fenómeno deportivo. Campos en Ciencias Sociales, 4(1), 101-136. http://dx.doi.org/10.15332/s2339-3688.2016.0001.05

Rivera, S. L. O. (2013). Lo comunitario en las radios comunitarias: Sentidos en juego. Campos en Ciencias Sociales, 1(1), 89-105. 
Rocha, L. F. Z. (2018). Políticas de habitabilidad en calle en Bogotá, Colombia ¿hacia el desarrollo humano integral? Campos en Ciencias Sociales, 6(1), 43-72. https://doi.org/10.15332/s2339-3688.2018.0001.02

Rodrigues, D. dos S. (2014). Memorias de la secularización: El registro de las transformaciones en el escenario religioso brasilero a través de las estadísticas de individuos identificados como sin religión. Campos en Ciencias Sociales, 2(1), 109138. https://doi.org/10.15332/s2339-3688.2014.0001.03

Rodríguez, A. S. (2013). El manejo informativo del desplazamiento forzado en dos medios electrónicos colombianos: Eltiempo.com y semana.com. Campos en Ciencias Sociales, 1(2), 251-278. https://doi.org/10.15332/s2339-3688.2013.0002.02

Rodríguez, A. T. C. (2014). Patrimonio y memoria: Encuentros y desencuentros por el hallazgo del cementerio muisca en la hacienda El Carmen, en Bogotá, D. C. Campos en Ciencias Sociales, 2(2), 207-223. https://doi.org/10.15332/s23393688.2014.0002.04

Rodríguez, C. A. L. (2013). Del ejercicio de la prostitución al ejercicio del trabajo sexual como forma de reivindicar derechos negados. Campos en Ciencias Sociales, 1(1), 169-196.

Rodríguez, D. E. A. (2019). Media Ecology. A Field of Study. Reflections about the beginning of the Doctoral Program in communication at Universidad de La Sabana. Campos en Ciencias Sociales, 7(2), 249-251. https://doi.org/10.15332/25006681.5130

Rodríguez, E. C. (2014). Lecciones de memoria. El recuerdo de 1971 en las protestas estudiantiles de 2011 en Colombia. Campos en Ciencias Sociales, 2(2), 287-314. https://doi.org/10.15332/s2339-3688.2014.0002.02

Rodríguez, J. A. R. (2013). Doscientos años de un periódico no tan feliz. Campos en Ciencias Sociales, 1(2), 373-386. https://doi.org/10.15332/s2339-3688.2013.0002.08

Rodríguez, J. S. B. (2016). Catedrales como bibliotecas: Filosofía, literatura y espiritualidad. Campos en Ciencias Sociales, 4(1), 59-80. http://dx.doi.org/10.15332/s2339-3688.2016.0001.03

Rojas, D. A. G. (2015). La co-creación como plataforma estratégica para reposicionar la marca ciudad. Caso de estudio: Medellín, ciudad innovadora. Campos en Ciencias Sociales, 3(2), 177-198. https://doi.org/10.15332/s2339-3688.2015.0002.03

Rojas, P. R., \& Yépez, J. G. (2014). Historia oral: ¿una historia popular? Campos en Ciencias Sociales, 2(1), 11-50. https://doi.org/10.15332/s2339-3688.2014.0001.05

Romero, C. B., Cediel, D. J. L., Romero, C. R., Moreno, M. R., \& Díaz, L. N. (2019). "Allá en La Bonga viví yo". Expresiones de memoria colectiva sobre los efectos del conflicto armado en Colombia. Campos en Ciencias Sociales, 7(1), 17-42. https://doi.org/10.15332/25006681.4523

Romero, J. H. C., \& Ospina, E. A. S. (2020). La construcción de paz a través de la consolidación del conocimiento biológico y territorial. Campos en Ciencias Sociales, 8(1), Article 1. https://doi.org/10.15332/25006681/5719

Rubiano, A. R. (2015). Jóvenes y representaciones sociales sobre la foto de perfil en Facebook. Campos en Ciencias Sociales, 3(2), 133-152. https://doi.org/10.15332/s2339-3688.2015.0002.01

Salas, R. D. (2013). Espacios comunicativos para la convivencia y la participación. Pensamiento y práctica desde la IAP. Campos en Ciencias Sociales, 1(1), 77-88.

Salazar, E. (2016). Muy bonito todo. Investigar imágenes y objetos contemporáneos desde la cultura mediática y la cultura popular. Campos en Ciencias Sociales, 4(2), 199-217. https://doi.org/10.15332/s2339-3688.2016.0002.02

Sánchez, C. A. D. (2019). "Hay que buscar medios con espacios neutros y de diálogo, en donde todos podamos opinar" Entrevista a Thomas Tufte, profesor de la Universidad de Leicester, Reino Unido. Campos en Ciencias Sociales, 7(1), 249-254.

Sarmiento, C. A. R. (2016). Heraldos de cine: Imágenes del cine y la construcción estética del colombiano moderno. Campos en Ciencias Sociales, 4(2), 167-197.

https://doi.org/10.15332/25006681.3467 
Soares, M. C. P., Caetano, A. M. S., \& Barbosa, M. R. (2020). Bringing Together Psychology and Peace: A Critique towards the Emancipatory Potential of Peace Psychology. Campos en Ciencias Sociales, 8(1), Article 1. https://doi.org/10.15332/25006681/5715

Suárez, D. A. A. (2017). Economía de la coca y violencia: Realidades desde el Corregimiento de El Plateado, Municipio de Argelia, Cauca (Colombia). Campos en Ciencias Sociales, 5(1 y 2), 39-72. https://doi.org/10.15332/s2339-3688.2017.0001.02

Tinel, F.-X. (2015). Desarrollo e hiperconsumo: La producción de lo efímero. Campos en Ciencias Sociales, 3(1), 79-96. https://doi.org/10.15332/s2339-3688.2015.0001.04

Uranga, W. (2015). Comunicación para el diálogo político e intercultural. Derecho a la comunicación y ciudadanía comunicacional. Campos en Ciencias Sociales, 3(1), 5178. https://doi.org/10.15332/s2339-3688.2015.0001.03

Urra, M. (2014) Responsabilidad social y medio ambiente. Algunas reflexiones desde el trabajo social. Memorias del Foro "Perspectivas ambientales en el mundo Contemporáneo", 88-91. Disponible en: https://www.researchgate.net/publication/270824050 Responsabilidad social y medi o ambiente Algunas reflexiones desde el trabajo social

Urra Canales, M. (2019). Análisis de la producción académica de la Facultad de Sociología de la Universidad Santo Tomás, desde los campos de acción de la misma universidad. https://doi.org/10.13140/RG.2.2.26949.60640

Urrea, L. C. (2013). Entre la participación ciudadana y las resistencias creativas: Una experiencia en la localidad de Engativá. Campos en Ciencias Sociales, 1(1), 43-76.

Valencia, A. M. S. (2020). Factores sociales, económicos y ambientales de las organizaciones de economía solidaria conformadas en situaciones de crisis en Colombia. Campos en Ciencias Sociales, 8(1), Article 1. https://doi.org/10.15332/25006681/5258

Vega, J. A. G. (2014). Reseña bibliográfica: Cartel ilustrado en Colombia: década 19301940. Campos en Ciencias Sociales, 2(1), 161-164.

Velásquez, F. R. (2016). Cine, poder e historia: La representación y construcción social del indígena en el cine ficción venezolano durante la década de los años 80. Campos en Ciencias Sociales, 4(1), 11-31. https://doi.org/10.15332/s2339-3688.2016.0001.01

Vélez, N. M., \& López, F. (2020). Lectura, escritura y oralidad: La narración colectiva de Colombia en tiempos de posconflicto. Campos en Ciencias Sociales, 8(1), Article 1. https://doi.org/10.15332/25006681/5721

Vieco, J. J. (2015). Los sistemas productivos tradicionales y el programa RESA en el resguardo Ticoya de Puerto Nariño. Campos en Ciencias Sociales, 3(1), 13-33. https://doi.org/10.15332/s2339-3688.2015.0001.01

Villegas, V. A. L. (2017). El conflicto, la guerra y la paz. Una aproximación desde la perspectiva del realismo político en Nicolás Maquiavelo. Campos en Ciencias Sociales, $5(1$ y 2), 137-157.

Zabala, O. A. G. (2017). Constitución y ampliación de resguardos indígenas en Colombia. Una mirada al avance histórico y perspectivas de cumplimiento. Campos en Ciencias Sociales, 5(1 y 2), 73-113. https://doi.org/10.15332/s2339-3688.2017.0001.03 